\title{
Studies of MicroMeGas Chambers for the New Small Wheel using Cosmic Muons
}

\author{
T. Klapdor - Kleingrothaus, U. Landgraf, N. Scheidtmann, S. Zimmermann
}

\begin{abstract}
Micromesh Gaseous Detectors (MicroMeGas) will be used in the ATLAS detector for the construction of the New Small Wheel Upgrade during the long shut down II in 2019/20. These detectors are used primarily for accurate position measurement but can also provide additionally trigger information. The spatial resolution is of the order of $100 \mu \mathrm{m}$. In parallel to the ongoing constructions of the actual size prototypes, additional performance studies with small MicroMeGas prototypes of a size of $10 \times 10 \mathrm{~cm}^{2}$ are performed. These studies include a cosmic muon test setup in combination with a scalable readout system, such that the effect of variations in the pressure of the operation gas or changes in the humidity at the lower ppm level to the detector performance are investigated. These parameters will have an impact on the final design of the slow control system at the New Small Wheel in ATLAS.
\end{abstract}

Index Terms-MicroMeGas, New Small Wheel, ATLAS

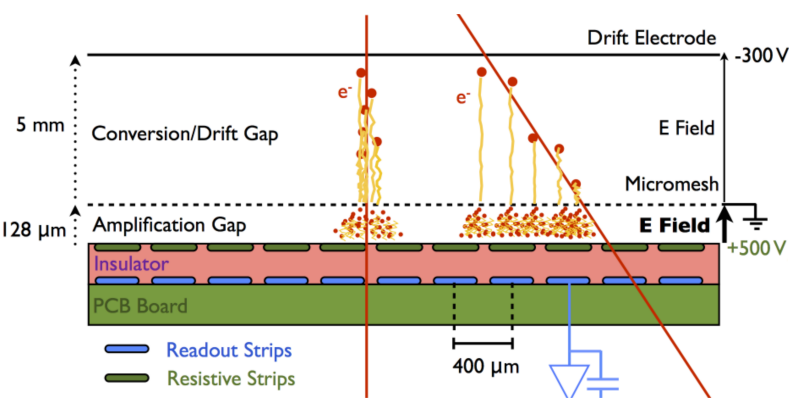

Fig. 1. Schematic cut of a MicroMeGas detector. The matching layer of resistive strips with the readout structure makes the detector insensitive to discharge from massive ionization and to sparking.

\section{Resistive Strip MicroMeGas Detectors}

\section{INTRODUCTION}

$\mathbf{M}$ ICROMESH Gaseous Detectors (MicroMeGas), which were introduced in 1996 by Giomataris [1], are now well established in particle physics. Their high rate capability in combination with a high intrinsic spatial resolution make them popular for High-Energy Physics experiments.

It is foreseen that the Large Hadron Collider (LHC) will be upgraded in two phases to increase the luminosity by a factor of 5. In order to deal with the higher rate, the ATLAS detector [2] has to be upgraded, too [3]. In 2019/20 during the second long shutdown of LHC the two innermost endcap stations (Small Wheels), which are part of the Muon Spectrometer and cover the high-pseudorapidity regions, will be replaced. The New Small Wheel (NSW) Upgrade program [4] of the ATLAS detector will use MicroMeGas technology to cover an active area of about $1280 \mathrm{~m}^{2}$. The NSW will have a similar geometry to the present Small Wheel, namely a disk-like one and will be segmented into 16 sectors, with a diameter of approximately $10 \mathrm{~m}$ [4]. Furthermore, two sets of small-strip Thin Gap Chambers (sTGC) with four layers each will be interlaid by these MicroMeGas detectors resulting in a fully redundant system. The NSW will be capable of operating at rates up to $15 \mathrm{kHz} / \mathrm{cm}^{2}$ and will add a trigger functionality to the first muon station.

The targeted performance of the NSW is to reach a spatial resolution of $100 \mu \mathrm{m}$ and an angular resolution of $\leq 1 \mathrm{mrad}$.

This work was carried out on behalf of the ATLAS Muon Collaboration.

The authors are with the Institute of Physics, Freiburg University, Germany (e-mail: klapdor-kleingrothaus@physik.uni-freiburg.de).

Manuscript received November 30, 2016
The gas volume of a typical MicroMeGas detector is divided into two regions: a drift region of several millimeters and an amplification region, which has typically a size of 100 to $150 \mu \mathrm{m}$ (see fig. 1).

A micromesh, which is woven of wires with a diameter of $\sim 20 \mu \mathrm{m}$ and a pitch of $\sim 64 \mu \mathrm{m}$, divides this volume into these two regions. The electric field at the drift region is usually $\sim 600 \mathrm{~V} / \mathrm{cm}$ and in the amplification region around 35 to $45 \mathrm{kV} / \mathrm{cm}$. For the NSW it is foreseen to use the gas mixture $93 \% \mathrm{Ar}$ and $7 \% \mathrm{CO}_{2}$ as operation gas. The large amplification factor of an order of $10^{4}$ is essential for the detection of minimum ionizing particles with a high efficiency.

In the drift and conversion region ionized molecules and electrons will be created by ionization through incoming charged particles. The electrons drift within several ns towards the mesh, which is transparent to more than $95 \%$ of the electrons. Reaching the thin amplification region an electron avalanche is created producing a fast pulse on the readout strips [5]. The process is sketched in fig. 1 for perpendicular and inclined tracks.

The resistive strip pattern, which matches the readout pattern is introduced to decrease the probability of discharges (sparks) which could occur due to the high field. Such discharges can lead to a high-voltage breakdown that could cause a large dead time and can damage the detector. The resistive strips reduce on one hand the signal amplitude but on the other hand allow an operation at a higher gas gain due to the by about three orders of magnitude reduced sparking probability [6] [7] [8]. 


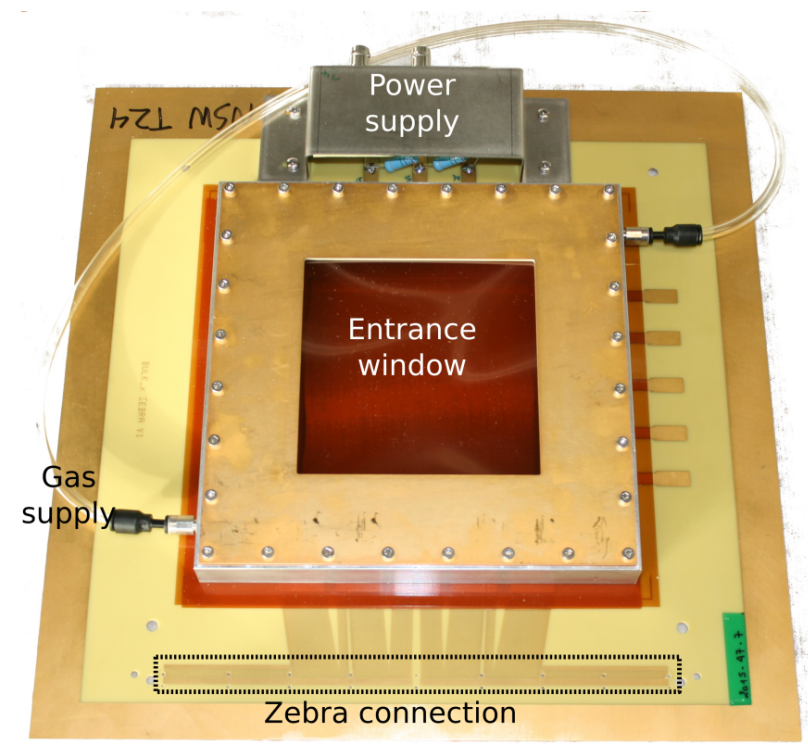

Fig. 2. T-Bulk MM detector with elastomeric connector layout.

\section{A. The T-Bulk MicroMeGas Chambers}

The RD51 collaboration at CERN [9] developed MicroMeGas detector prototypes, so called T-Bulk MM detectors, to test the suitability of this technology for the NSW purpose. These prototypes (see fig. 2), which are studied here, have a dimension of $10 \times 10 \mathrm{~cm}^{2}$ and are connected to the readout electronics via an elastomeric connector which is commercially available and also known as ZEBRA connector [10]. These connectors will be used for the final detector at the NSW as well. The amplification region has a height of $128 \mu \mathrm{m}$ which is separated by a micromesh from the drift and the conversion region with a height of $\sim 5 \mathrm{~mm}$. The wires of this mesh have a diameter of $18 \mu \mathrm{m}$ and a pitch of $63.5 \mu \mathrm{m}$. The readout strips have a width of $150 \mu \mathrm{m}$ and a pitch of $250 \mu \mathrm{m}$. In the drift region a field of $\sim 600 \mathrm{~V} / \mathrm{cm}$ and in the amplification region a field of $\sim 42 \mathrm{kV} / \mathrm{cm}$ is applied.

\section{The Cosmic Muon Test Setup}

The cosmic muon test setup in Freiburg (see fig. $3 \& 4$ ) operates two T-Bulk MicroMeGas chambers in parallel one on top of the other. The MicroMeGas detectors are closely stacked between two scintillators of a size of $15 \times 15 \mathrm{~cm}^{2}$ overlapping the $10 \times 10 \mathrm{~cm}^{2}$ active area of the detectors, to provide the trigger signal. The trigger signal is processed via NIM electronics before it reaches the readout electronics. The operation gas $\mathrm{Ar} / \mathrm{CO}_{2}$ (93/7) is provided by an in-house gas mixing system and the humidity can be regulated between $\sim 0$ to $3000 \mathrm{ppm}$.

\section{A. Readout Electronics}

The readout system is based on the Scalable Readout System (SRS) which was developed by the RD51 collaboration at CERN [9]. By using an adapter board it is possible to connect the APV25 modules (part of the SRS) to the elastomeric readout pattern of the MicroMeGas detectors. A typical muon signal from this readout chain is shown in fig. 5 recorded with

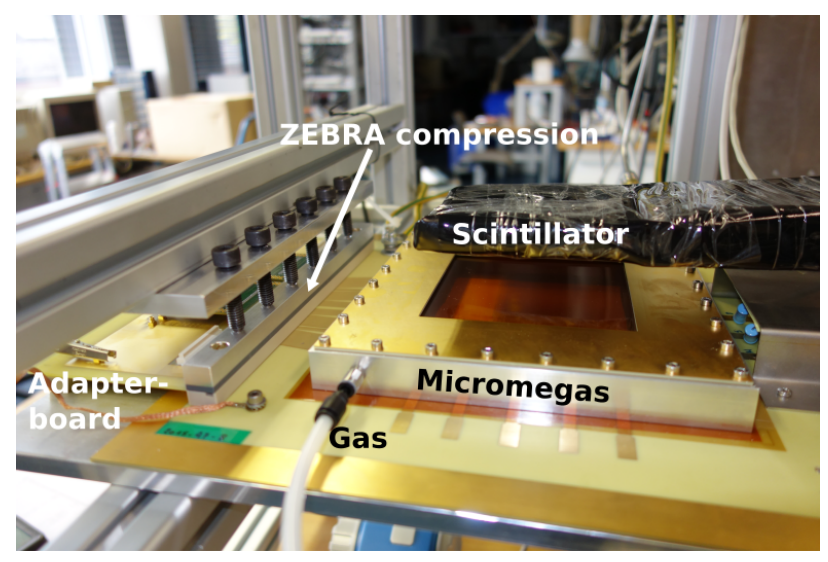

Fig. 3. Cosmic muon test setup: Upper T-Bulk MM detector with continuous elastomeric connector compression system.

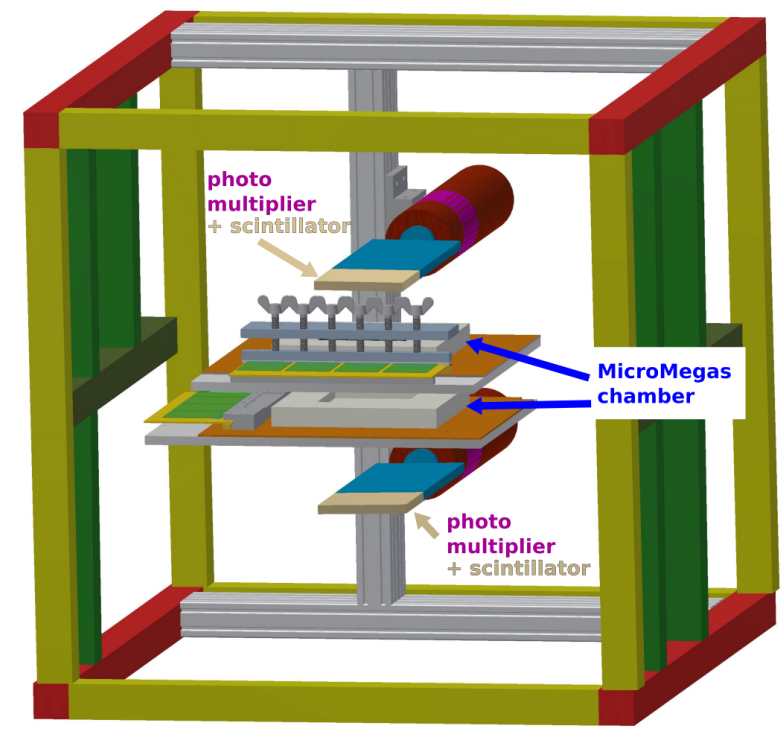

Fig. 4. The cosmic muon test setup (schematic).

the mmdaq software [11]. The mmdaq software was developed by the MAMMA collaboration as a data acquisition software for MicroMeGas detectors without assuming specific front-end readout electronics and offering the user a GUI for configuring and data taking.

\section{B. Elastomeric Connectors}

An elastomeric connector provides high-density redundant electrical paths for high-reliability connections. Their benefits are their flexibility, their radiation hardness, their compact dimension and that the soldering of connectors can be avoided. Thus, they are chosen to connect the readout strips of the MicroMeGas detector with the front-end electronics. To readout the MicroMeGas prototypes as well as detectors at the NSW elastomeric connectors of the dimensions $\mathrm{H} \times \mathrm{B} \times \mathrm{L}$ : $\sim 6.4 \times 3 \times 105 \mathrm{~mm}^{3}$ are used. For a proper connection of these connectors a compression force of $\sim 150 \mathrm{~N}$ is necessary as well as a precise mapping of the readout pattern of less than $100 \mu \mathrm{m}$. In addition to the already existing discrete 


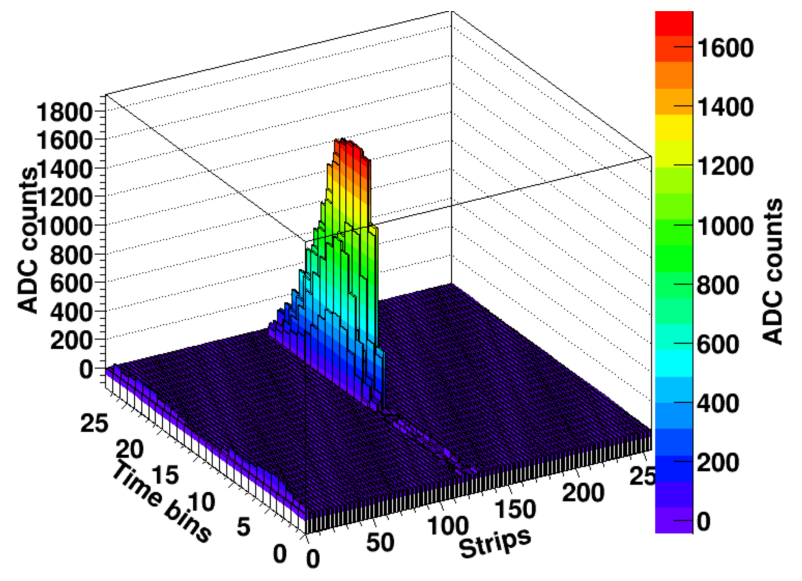

Fig. 5. Typical signal of a muon in a T-Bulk MM detector.

compression system for the NSW, which is used at the lower T-Bulk MM detector, a continuous system was developed and is used at the upper T-Bulk MM detector.

\section{TARGETED EXPERIMENTS}

With the setup described above, the working point of the MicroMeGas detector prototypes will be studied focusing on the slow control of the high-voltage power supply of the NSW detector system, where it is necessary to react properly to changes of gas parameters (pressure, temperature, ...) to ensure a stable operation. Furthermore, the effect of humidity in this gas will be studied at the lower ppm level. These studies are going on presently in Freiburg.

\section{T-Bulk Measurements}

In the following the pressure dependence of the gain on basis of measurements and simulation is discussed. Furthermore, the first simulation results for different amounts of air in the operation gas are presented.

\section{A. Voltage and Pressure Dependence}

The electron multiplication (gain) of a gaseous detector depends strongly of the characteristics of the operation gas as well as on the applied electrical field $E$. The gain in a uniform electric field between two parallel plates with a distance $d$ in a gas with pressure $\mathrm{p}$ is given by: $G=\exp (\alpha(p, E) \cdot d)$. The first Townsend coefficient $\alpha(p, E)$ describes the mean free path of an electron between two ionization processes. An approximation of the Townsend coefficient $\alpha(p, E)$ is given by Rose and Korff [13]: $\alpha(p, E)=p \cdot A \cdot \exp \left(-B \frac{p}{E}\right)$. Thus, the gain is given by

$$
G=\exp \left(d \cdot p \cdot A \cdot \exp \left(-B \cdot \frac{p}{E}\right)\right) .
$$

$\mathrm{A}$ and $\mathrm{B}$ are dependent on the gas mixture.

The Diethorn formula [14] [15] describes the dependence of the gain on the pressure of the gas and the amplification voltage for cylindrical gas detectors:

$$
G=\frac{U}{r_{a} \cdot \ln \left(\frac{r_{c}}{r_{a}}\right) \cdot E_{\min }\left(p_{0}\right) \cdot \frac{p}{p_{0}}} \exp \left(\frac{U \cdot \ln 2}{\frac{r_{c}}{r_{a}} \cdot \Delta V}\right)
$$

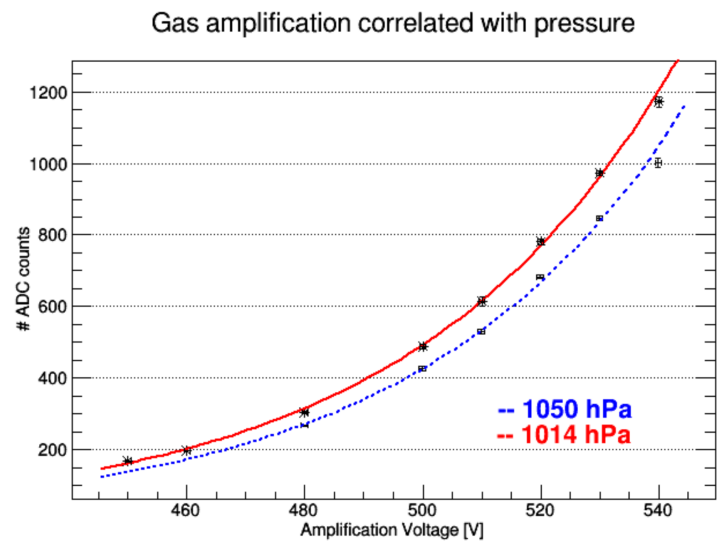

Fig. 6. Determination of the gain of the T-Bulk MM detector with respect to the amplification voltage at two different pressure values.

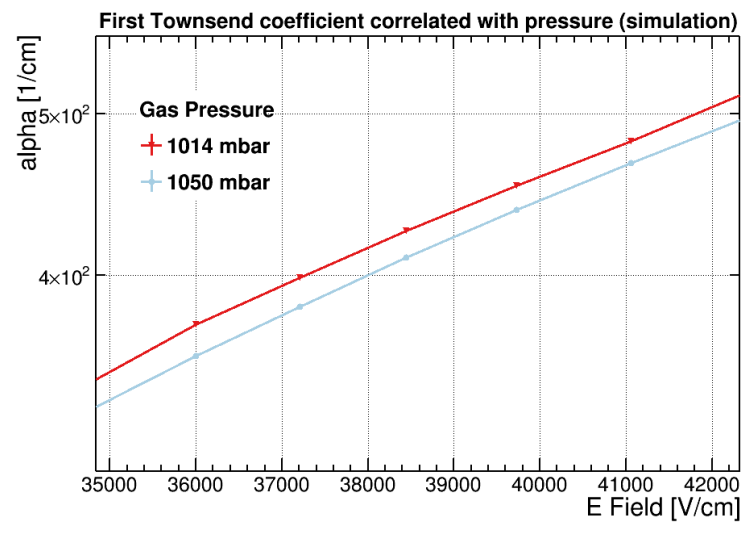

Fig. 7. MAGBOLTZ simulation of the first Townsend coefficient for $\mathrm{Ar} / \mathrm{CO}_{2}$ (93/7) at different pressures.

The gain increases exponentially with the amplification voltage and is inversely proportional to the pressure.

At the cosmic muon setup, the pressures of $1014 \mathrm{mbar}$ and $1050 \mathrm{mbar}$ were chosen and the response was measured for an amplification voltage between 480 to $540 \mathrm{~V}$, which corresponds to an electric field between $\sim 35$ to $42 \mathrm{kV} / \mathrm{cm}$. The effects described by the above formula can be seen with the T-Bulk MM detector as shown in fig. 6.

\section{B. Simulation of the Gas Amplification}

MAGBOLTZ [12] was used to simulate the first Townsend coefficient for a gas mixture of $\mathrm{Ar} / \mathrm{CO}_{2}$ assuming neither humidity nor air for both pressure values, which were used in the experiment. As it is shown in fig. 7 the Townsend coefficient for a pressure of $1050 \mathrm{mbar}$ is lower than the ones for 1014 mbar. The gain can be calculated from the first Townsend coefficient, but different detector parameters have to be considered which are not determined, yet. However, the ratio of the slopes provides a reasonable parameter to compare the result from the simulation with the measurements. Exponential fits were performed for the data as well as for the simulation. The determined values for the slopes are summarized in table I. The ratio for the measurements is determined to 1.011(15) while for the simulation the ratio 


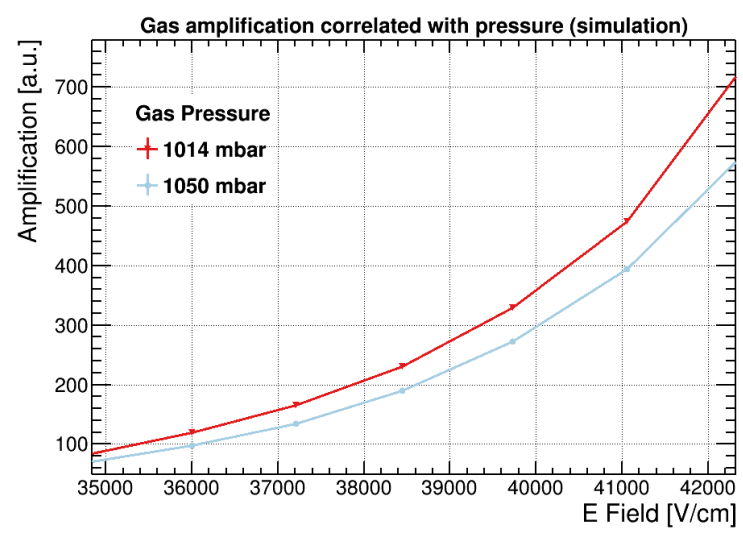

Fig. 8. Calculation of the gain $G=\left.\exp (\alpha \cdot x)\right|_{x=128 \mu \mathrm{m}}$ using the MAGBOLTZ simulated Townsend coefficient $\alpha$ from fig. 7 .

\begin{tabular}{llll}
\hline & 1014 mbar & 1050 mbar & Ratio \\
\hline Slope (exp.) & $22.58(30) \cdot 10^{-4}$ & $22.34(17) \cdot 10^{-4}$ & $1.011(15)$ \\
Slope (sim.) & $28.71 \cdot 10^{-5}$ & $28.50 \cdot 10^{-5}$ & 1.007 \\
\hline
\end{tabular}

TABLE I

FIT VALUES ON THE AMPLIFICATION MEASURED IN THE EXPERIMENT AND DETERMINED IN THE SIMULATION.

is calculated to 1.007. As a first approximation these values show a good agreement between measurement and simulation. More studies concerning pressure changes and humidity are in progress.

\section{Humidity and Air in $\mathrm{Ar} / \mathrm{CO}_{2}$}

The developed gas supply system for the MicroMeGas at the cosmic muon setup allows to add well-defined amounts of humidity to the operating gas. The humidity in the gas is monitored with a dew point meter, which can determine a humidity down to $\sim 0.14 \mathrm{~g} / \mathrm{m}^{3}$. Measurements are ongoing to study the change of the gain in dependence of humidity and pressure at different amplification voltages.

Following the humidity studies it is foreseen to measure the effect of air in the gas mixture. Fig. 9 shows the change of the first Townsend coefficient, which is calculated with MAGBOLTZ, as function of the electrical field considering different amounts of air by a given humidity. The negative effect of air to the Townsend coefficient and consequently to the gain is increasing with an increasing fraction of air. In this example the Townsend coefficient decreases by $1.32 \%$ by a fraction of air of $0.01 \%$ while for $1 \%$ air is decreased by $3.01 \%$ considering an electric field of $38 \mathrm{kV}$. This indicates that in the measurements small variations of the gain have to be determined.

\section{CONCLUSion AND PRospects}

In this work a setup is described, which has been installed in Freiburg and allows to operate two T-Bulk MM detectors in series using cosmic muons. The setup allows to change the pressure of the operation gas and to add defined amounts of humidity in order to study the effects on the performance of the

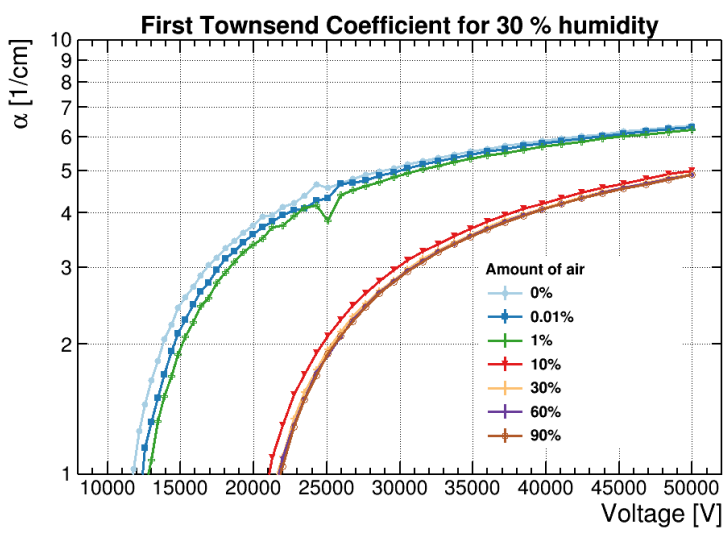

Fig. 9. First Townsend coefficient for the operating gas $\mathrm{Ar} / \mathrm{CO}_{2}$ with admixed air by a fixed humidity. With increasing fraction of air, the Townsend coefficient decreases and will result in less amplification. The data was simulated with MAGBOLTZ.

detector. The simulation tool MAGBOLTZ was used and the obtained results were compared to the measured data. Both are in good agreement which motivates for the ongoing detailed simulation of the detector as well as for ongoing measurements with different humidity and pressure values. These studies shall be used to give recommendations with respect to the working point for the MicroMeGas to be used in the New Small Wheel of the ATLAS detector, which will be installed in $2019 / 20$.

\section{ACKNOWLEDGMENT}

The authors would like to thank the RD51 collaboration, the MAMMA collaboration and the LMU Munich for their support in operating the T-Bulk MM detectors. Special thanks to André Zibell of the LMU Munich for providing a configuration tool for the SRS system.

\section{REFERENCES}

[1] Y. Giomataris et al., MICROMEGAS: A High granularity position sensitive gaseous detector for high particle flux environments, Nucl. Instrum. Meth. A376, 29-35, (1996)

[2] G. Aad et al. [ATLAS Collaboration], JINST 3, S08003 (2008). doi:10.1088/1748-0221/3/08/S08003

[3] ATLAS collaboration, Letter of Intent for the Phase-I Upgrade of the ATLAS Experiment, CERN-LHCC-2011-012. LHCC-I-020, 2011

[4] T. Kawamoto et al., New Small Wheel Technical Design Report, ATLASTDR-20-2013, CERN-LHCC-2013-006, 2013

[5] I. Giomataris et al., MicroMeGas in a bulk, Nucl.Instrum.Meth. A560, 405-408, (2006)

[6] T. Alexopoulos et al., A spark-resistant bulk-micromegas chamber for high-rate applications, Nucl. Instr. Meth. A 640 110-118 (2011).

[7] T. Alexopoulos et al., Using Micromegas in ATLAS to Monitor the Luminosity, ATL-MUON-PUB-2013-002 (2013).

[8] Y. Kataoka et al., Performance studies of a micromegas chamber in the ATLAS environment, JINST 9 C03016 (2014).

[9] RD51 collaboration, http://rd51-public.web.cern.ch/RD51-Public/ Activities/Documents/WG5SRS.pdf

[10] Fujipoly, ZEBRA Elastomeric Connectors www.fujipoly.com

[11] M. Bianco et al., Development and test of a versatile DAQ system based on the ATCA standard, Proceedings of Technology and Instrumentation in Particle Physics 2014 (TIPP2014). 2-6 June 2014. Amsterdam

[12] Magboltz - Transport of electrons in gas mixtures, http://magboltz.web. cern.ch/magboltz/

[13] Y. Giomataris, Nucl. Instr. Meth. A419, 239-250, (1998) 
[14] W. Diethorn, A methane proportional counter system for natural radiocarbon measurements, USAEC Report NY06628 (1956); Carnegie Inst. of Technology, 1956

[15] R. Wolff, Measurement of the gas constants for various proportionalcounter gas mixtures, Nucl. Instrum. Meth 115,2 461 - 463, (1974) 